\title{
Molecular Interaction Studies in Binary Liquid Mixtures from Ultrasonic Data
}

\author{
N.SANTHI*, PL.SABARATHINAM ${ }^{\#}$, M.EMAYAVARAMBAN \\ C.GOPI and C.MANIVANNAN
}

Department of Chemistry,

Government Arts College, Chidambaram, Tamilnadu, India.

"Departmental of Chemical Enginering,

Annamalai University,Annamalai Nagar - 608002, Tamilnadu, India.

emayavarambanm@gmail.com

Received 20 July 2009; Accepted 15 September 2009

\begin{abstract}
Ultrasonic velocities and densities of the binary liquid mixtures of dimethy1 sulphoxide (DMSO) with phenol, $o$-cresol, $m$-cresol, $p$-cresol and $p$-chlorophenol at $318.15 \mathrm{~K}$, over the entire composition range were measured. The theoretical values of ultrasonic velocity were evaluated using the Nomoto's Relation (NR), Ideal Mixture Relation (IMR), Free Length Theory (FT) and Collision Factor Theory (FLT). The validity of these relations and theories was tested by comparing the computed sound velocities with experimental values. Further, the molecular interaction parameter $(\alpha)$ was computed by using the experimental and the theoretical ultrasonic velocity values. The variation of this parameter with composition of the mixtures has been discussed in terms of molecular interaction in these mixtures.
\end{abstract}

Keywords: Ultrasonic velocities, Inter molecular interactions, Binary Mixtures.

\section{Introduction}

Measurements of ultrasonic velocity in liquids ${ }^{1-5}$ give information about physicochemical behaviour of liquid mixtures such as molecular association and dissociation. Ramasawamy and Anbananthan ${ }^{6}$ carried out the ultrasonic investigations on some binary and ternary liquid mixtures and correlated the experimental findings of ultrasonic velocity with the theoretical relations suggested by Nomoto ${ }^{7}$ and van Deal van Geel $^{8,9}$ and interpreted the results in terms of molecular interactions. Mishra and Mishra ${ }^{10}$ have also studied the ultrasonic velocity of liquid mixtures using both the above relations and discussed the applicability of the above relations. 
In the present communication the Nomoto's Relation, Ideal Mixture Relation, Free Length and Collision Factor theories have been used to predict ultrasonic velocity in Phenols with DMSO liquid mixtures and they have been compared with the experimental values of ultrasonic velocity. Further, the intermolecular interaction has been discussed in the light of deviations observed by plotting the experimental and the theoretical values of ultrasonic velocity against the mole fraction of the pheno1[s].

\section{Experimental}

Ultrasonic velocity of pure liquids and mixtures were measured at $3 \mathrm{MHz}$ with variable path single beam interferometer gold plated quartz crystal at the bottom and double walled gold plated cell having grooves at inside walls to check the overlapping of stationary waves to produce clarity nodes and inter codes. The accuracy of velocity measurements is $\pm 0.1 \mathrm{~ms}^{-1}$. The densities of pure liquids and mixtures were determined from the weight measurements on using the specific graving bottle immersed in the thermostat at the experimental temperature and the accuracy in the measurements ${ }^{11,12}$ was $\pm 0.1 \mathrm{kgm}^{3}$.

The liquids were of best available grade phenol (BDH), $o$-cresol (SD's fine chemicals), $m$-cresol (BDH), $p$-cresol (E Merck), dimethyl formamide (BDH) and purified by standard methods described by weissberger. The liquid mixtures were prepared by mixing calculated amount of pure liquids.

\section{Theoretical aspects}

Nomoto established an empirical formula for ultrasonic velocity in binary liquid mixture,

$$
U_{N M}=\left[\left(x_{1} R_{1}+x_{2} R_{2}\right) /\left(x_{1} V_{1}+x_{2} V_{2}\right)\right]^{3}
$$

Where, $\mathrm{U}_{\mathrm{NM}}=$ ultrasonic velocity as per Nomoto, $\mathrm{R}=\mathrm{Rao}$ constant, is related to molecular weight $\mathrm{M}$ and density $\rho$ by,

$$
R=(M / \rho) U^{1 / 3}=V_{M} U^{1 / 3}
$$

Where, $\mathrm{V}_{\mathrm{M}}$ molar volume obey the additivity, $V_{M}=x_{1} V_{1}+x_{2} V_{2}$

Van Deal and Van Geel proposed the following expression for the estimation of sound velocity $\mathrm{u}_{\mathrm{im}}$ in an ideal mixture using the sound velocities in the pure components

$$
u_{i m}=\left[1 /\left(x_{1} M_{1}+x_{2} M_{2}\right)^{1 / 2}\right]\left\{1 /\left[\left(x_{1} / M_{1} u_{1}{ }^{2}\right)+\left(x_{2} / M_{2} u_{2}{ }^{2}\right)\right]^{1 / 2}\right\}
$$

According to Jacobson's theory ${ }^{13}$ of free length, the ultrasonic velocity is given by

$$
u=K /\left(L_{f} \rho^{1 / 2}\right)=K Y /\left(2 V_{a} \rho^{1 / 2}\right)
$$

For the Binary liquid mixture Eq. (4) is

$$
u=K\left(x_{1} Y_{1}+x_{2} Y_{2}\right) / 2\left[V_{M}-\left(x_{1} V_{0,1}+x_{2} V_{0,2}\right)\right] \rho^{1 / 2}
$$

$\mathrm{Y}$ as an adjustable parameter in the evaluation of velocity in liquid mixtures. $\mathrm{Y}$ was obtained from velocity in pure liquids using equation Eq.(4):

$$
Y=2 V_{a} U \rho^{1 / 2} / K
$$

where $\mathrm{K}$ is the temperature - dependent Jacobson's constant ${ }^{14,} \mathrm{~V}_{\mathrm{a}}$ is the available molar volume, which is the difference between the molar volumes at $\mathrm{T}(\mathrm{K})$ and $0(\mathrm{~K})$, is a direct measure of the compactness and the strength of bonding between the molecules of a liquid in mixture. $\mathrm{V}_{\mathrm{a}}$ is given by

$$
\mathrm{V}_{\mathrm{a}}=\mathrm{V}_{\mathrm{M}}-\mathrm{V}_{0}
$$


when $\mathrm{Y}$ in Eq. (5) is replaced according to Eq. (6) and when the resulting expression is rearranged, one gets

$$
\left.U_{(F L T)}=\left\{x_{1}\left(V_{M 1}-V_{0,1}\right) U_{1} \rho_{1}{ }^{1 / 2}\right]+\left\lfloor x_{2}\left(V_{M 2}-V_{0,2}\right) U_{2} \rho_{2}{ }^{1 / 2}\right]\right\} /\left[V_{M}-\left(x_{1} V_{0,1}+x_{2} V_{0,2}\right)\right] \rho^{1 / 2}
$$

Eq. (8) says that according to the free length theory the square root of the inverse of the adiabatic compressibility of liquid mixture $\left(\mathrm{U} \rho^{1 / 2}\right)$ is the sum of the available volume fraction average of the square root of the inverse of adiabatic compressibilities of the individual components.

\section{Collision factor theory (CFT)}

According to Schaaffs ${ }^{15}$ the sound velocity in pure liquid is given by

$$
U=U_{\infty} S B / V_{M}
$$

where $\mathrm{U}_{\infty}=1600 \mathrm{~m} / \mathrm{s} ; \mathrm{S}=$ collision factor and $\mathrm{B} / \mathrm{V}_{\mathrm{M}}=$ space filling factor, $\mathrm{B}$ is the actual volume of molecules per mole.

For binary liquid mixtures this formula was modified by Nutsch - Kuhnekies $^{16}$ as:

$$
\mathrm{U}_{(\mathrm{CFT})}=\left(\mathrm{U}_{\infty} / \mathrm{V}\right)\left[\left(\mathrm{x}_{1} \mathrm{~S}_{1}+\mathrm{x}_{2} \mathrm{~S}_{2}\right)\left(\mathrm{x}_{1} \mathrm{~B}_{1}+\mathrm{x}_{2} \mathrm{~B}_{2}\right)\right]
$$

$\mathrm{B}=\mathrm{b} / 4$, where $\mathrm{b}$ is the van der Waals parameter given by

$$
\mathrm{B}=(\mathrm{M} / \rho)\left[1-\left(\mathrm{RT} / \mathrm{MU}^{2}\right)\left\{\left(1+\mathrm{MU}^{2} / 3 \mathrm{RT}\right)^{1 / 2}-1\right\}\right]
$$

$\mathrm{s}$ as an adjustable parameter, obtained from velocities in pure components.

$$
\mathrm{s}=(\mathrm{V} / \mathrm{B})\left(\mathrm{U} / \mathrm{U}_{\infty}\right)
$$

The degree of intermolecular interaction (molecular association) is given by,

$$
\alpha=\left(\mathrm{U}_{\exp }^{2} / \mathrm{U}_{\mathrm{im}}^{2}\right)-1
$$

\section{Results and Discussion}

Table 1 presents the experimental and computed ultrasonic velocity using NR - Eq. (4), IMR - Eq. (5), FLT - Eq. (8) and CFT - Eq. (10) and also the percentage relative deviation between experimental predicted ultrasonic velocity values for the Phenol[s] + DMSO systems at $318.5 \mathrm{~K}$ respectively.

The predictive abilities of various ultrasonic theories depend upon the strength interaction prevailing in a system; these theories generally fail to predict accurately the ultrasonic velocities where strong interactions supposed to exist and the average absolute percentage relative deviation is small in systems where the interactions are less or nil. The theoretically found ultrasonic velocities of liquid mixtures reveal that CFT shows better agreement than the other relations. Rajendran ${ }^{17}$ also arrived at the same conclusion.

The CFT consider that the elastic nature of spherical molecules is responsible for the molecular interaction. It is more valid in these mixtures. On the other hand, the FLT assumes that molecules are rigid spheres with no interaction between them and it is not valid in the present cases. Hence the deviation from the experimental ultrasonic velocity values is maximum in the FLT. Though both the IMR and NR fit the data reasonably well, the gives a better fit.

The average absolute percentage relative deviation values are maximum for the $p$-chlorophenol + DMSO systems (Table 1) in respect of two relations and two theories and minimum for the $o$-cresol + DMSO systems in respect of the relations and theories excluding the IMR. In case of the IMR, phenol + DMSO system exhibit minimum deviation. These results not only show the feasibility of these theories but also envisage the rising deviation with increasing nonideality in the mixture ${ }^{18}$. 
Table 1. Experimental and predicted ultrasonic velocity and their percentage deviation and degree of molecular interaction for the dimethyl sulphoxide with phenol, $o$-cresol, $p$ cresol, $m$ - cresol and $p$-chconophenol systems at $318.15 \mathrm{~K}$.

\begin{tabular}{|c|c|c|c|c|c|c|c|c|c|c|}
\hline \multirow{2}{*}{$\begin{array}{l}\text { Mole } \\
\text { fraction } X_{1}\end{array}$} & \multirow[t]{2}{*}{ Uexp $\mathrm{ms}^{-1}$} & \multirow[t]{2}{*}{ NR } & \multirow[t]{2}{*}{ IMR } & \multirow[t]{2}{*}{ FLT } & \multicolumn{6}{|c|}{ Absolute Percentage } \\
\hline & & & & & CFT & NR & IMR & FLT & CFT & $\alpha$ \\
\hline 1 & 2 & 3 & 4 & 5 & 6 & 7 & 8 & 9 & 10 & 11 \\
\hline \multicolumn{11}{|c|}{ Phenol - Dimethy1 sulphoxide } \\
\hline 0.0000 & 1426.5 & & & & & & & & & \\
\hline 0.10 & .5 & 1428.1 & 1425.4 & 1451.9 & 1432.1 & 0.31 & 0.50 & 1.36 & 0.03 & 0.0100 \\
\hline 0.2034 & 20 & 1429.7 & 1424.8 & 1470.6 & 1436.5 & 0.67 & 1.01 & 2.17 & 0.20 & 0.0206 \\
\hline 0.25 & 1 & 1431.1 & 1424.8 & 1488.2 & 1440.6 & 1.18 & 1.61 & 2.76 & 0.53 & 0.0331 \\
\hline 0.3963 & 57.2 & 1432.5 & 1425.3 & 1502.1 & 1444.0 & 1.69 & 2.19 & 3.08 & 0.90 & 0.0452 \\
\hline 0.4963 & 1465.2 & 1433.9 & 1426.4 & 1516.8 & 1447.7 & 2.14 & 2.65 & 3.52 & 1.20 & 0.0552 \\
\hline 0.5940 & 1461.2 & 1435.2 & 1427.9 & 1520.7 & 1449.4 & 1.78 & 2.28 & 4.07 & 0.80 & 0.0472 \\
\hline & & 1436.3 & 1429.7 & 1510.3 & 1448.8 & 1.30 & 1.75 & 3.79 & 0.44 & \\
\hline 0. & & 1 & 1434.7 & 1477.2 & 1445.2 & 0.42 & 0.68 & 2.26 & 0.05 & 137 \\
\hline & 6 & 1439.3 & 1437.5 & 1458.6 & 1442.7 & 0.23 & 0.36 & 1.11 & 0.01 & 0.0072 \\
\hline & 1440.0 & & & & & & & & & \\
\hline \multirow{2}{*}{\multicolumn{11}{|c|}{ 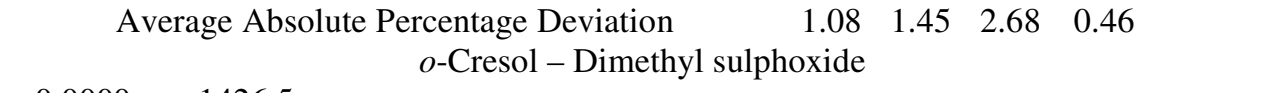 }} \\
\hline & & & & & & & & & & \\
\hline 0 & 1426.5 & & & & & & & & & \\
\hline & & 1429.9 & 1 & 5 & 1429.6 & 0.11 & 0.73 & 0.21 & 0.06 & 0.0148 \\
\hline 0 . & & 14 & 1416.2 & 1440.6 & 1432.4 & 0.18 & 1.20 & 0.50 & 0.07 & 0.0244 \\
\hline 0 . & 1 & 1 & 1414.1 & 1449.0 & 1435.2 & 0.42 & 1.70 & 0.72 & 0.24 & 0.0349 \\
\hline 0.4 & 1446.22 & 1434.3 & 1413.5 & 1456.9 & 1438.0 & 0.82 & 2.26 & 0.74 & 0.57 & 0.0468 \\
\hline & & 14 & 1414.3 & 1463.0 & 1440.2 & 1.10 & 2.57 & 0.78 & 0.79 & \\
\hline & & & 0 & 147 & 14 & 0.83 & 2.22 & 1.56 & 0.43 & 0. \\
\hline 0 & 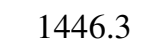 & 14 & 20.7 & 1466.8 & 1443.2 & 0.54 & 1.77 & 1.42 & 0.21 & 0.0363 \\
\hline & & 1439.7 & 1426.4 & 1455.3 & 1442.3 & 0.31 & 1.24 & 0.77 & 0.13 & 0.0252 \\
\hline 0. & 1443.5 & 1440.8 & 1433.8 & 1448.3 & 1442.1 & 0.18 & 0.67 & .33 & 0.10 & 0.0136 \\
\hline & 1441.8 & & & & & & & & & \\
\hline \multirow{3}{*}{\multicolumn{10}{|c|}{$\begin{array}{l}\text { Average Absolute Percentage Deviation } \\
m \text {-Cresol - Dimethyl sulphoxide } \\
0 \quad 1426.5\end{array}$}} & \\
\hline & & & & & & & & & & \\
\hline & & & & & & & & & & \\
\hline 0 . & & 1426.5 & .7 & 1444.3 & 1430.0 & 0.28 & 0.76 & 0.97 & 0.03 & 0.0153 \\
\hline 0.1997 & 5 & 1426.5 & 1414.5 & 1457.0 & 1432.5 & 0.56 & 1.39 & 1.57 & 0.14 & 0.0285 \\
\hline 0.3124 & 1440.6 & 1426.5 & 1410.4 & 1468.3 & 1434.7 & 0.98 & 2.09 & 1.92 & 0.41 & 0.0432 \\
\hline & & & 1408.4 & 1480.0 & 1436.8 & 1.53 & 2.78 & 2.17 & 0.82 & 0.0579 \\
\hline 0.499 & 145 & 1426.4 & 1407.8 & 1485.9 & 1437.7 & 1.84 & 3.12 & 2.25 & 1.06 & 0.0655 \\
\hline 0.6123 & 1447.6 & 1426.4 & 1408.7 & 1490.6 & 1438.4 & 1.46 & 2.68 & 2.97 & 0.63 & 0.0559 \\
\hline 0.7103 & 1440.3 & 1426.4 & 1411.1 & 1478.3 & 1436.3 & 0.96 & 2.03 & 2.64 & 0.28 & 0.0419 \\
\hline 0.7980 & 1435.2 & 1426.4 & 1414.3 & 1461.3 & 1433.2 & 0.61 & 1.45 & 1.82 & 0.14 & 0.0297 \\
\hline 0.9112 & 1430.8 & 1426.4 & 1420.3 & 1443.4 & 1429.8 & 0.31 & 0.73 & 0.88 & 0.07 & 0.0148 \\
\hline 1.0000 & 1426.4 & & & & & & & & & \\
\hline \multicolumn{6}{|c|}{ Average Absolute Percentage Deviation } & 0.95 & 1.89 & 1.91 & 0.40 & \\
\hline
\end{tabular}




\begin{tabular}{|c|c|c|c|c|c|c|c|c|c|}
\hline \multicolumn{10}{|c|}{$p$-Cresol - Dimethyl sulphoxide } \\
\hline 0.0000 & 1426.5 & & & & & & & & \\
\hline 0.1001 & 1429.5 & 1427.11420 .0 & 1437.4 & 1429.2 & 0.17 & 0.66 & 0.56 & 0.02 & 0.0134 \\
\hline 0.1950 & 1433.2 & 1427.71415 .4 & 1445.0 & 1431.2 & 0.38 & 1.24 & 0.82 & 0.14 & 0.0253 \\
\hline 0.2992 & 1438.2 & 1428.21411 .9 & 1454.7 & 1433.5 & 0.71 & 1.85 & 1.13 & .35 & 0.0381 \\
\hline 0.3964 & 1445.2 & 1428.71410 .1 & 1462.0 & 1435.2 & 1.14 & 2.43 & 1.16 & 0.69 & 0.0504 \\
\hline 0.4992 & 1454.0 & 1429.21409 .7 & 1469.7 & 1436.9 & 1.71 & 3.05 & 1.08 & 1.18 & 0.0638 \\
\hline 0.5887 & 1447.8 & 1429.61410 .7 & 1479.2 & 1438.8 & 1.26 & 2.56 & 2.17 & 0.62 & 0.0533 \\
\hline 0.6889 & 1442.5 & 1429.91413 .2 & 1469.4 & 1437.4 & 0.87 & 2.03 & 1.87 & 0.36 & 0.0419 \\
\hline 0.7899 & 1438.2 & 1430.31417 .3 & 1456.1 & 1435.2 & 0.55 & 1.45 & 1.24 & 0.21 & 297 \\
\hline 0.8 & 1434.5 & 1430.71423 .5 & 1444.0 & 1433.2 & 0.27 & 0.76 & 0.66 & .09 & 0.0155 \\
\hline 1.0000 & 1431.0 & & & & & & & & \\
\hline \multicolumn{5}{|c|}{ Average Absolute Percentage Deviation } & 0.78 & 1.78 & 1.19 & 0.41 & \\
\hline \multicolumn{10}{|c|}{$p$-Chlorophenol - Dimethyl sulphoxide } \\
\hline 0.0000 & 1426.5 & & & & & & & & \\
\hline 0.1102 & 1428.3 & 1422.01407 .0 & 1459.4 & 143 & 0.44 & 1.49 & 2.18 & 0.14 & 0 . \\
\hline 0.2138 & 1431.2 & 1418.21392 .8 & 1475.5 & 1431.0 & 0.91 & 2.68 & 3.10 & 0.02 & 58 \\
\hline 0.3121 & 1435.2 & 1414.81382 .8 & 1495.1 & 1431.9 & 1.42 & 3.65 & 4.17 & 0.23 & 0.0772 \\
\hline 0.4196 & 1440.3 & 1411.41375 .5 & 1504.5 & 1430.8 & 2.00 & 4.50 & 4046 & 0.66 & 0.0965 \\
\hline 0.5101 & 1443.5 & 1408.81372 .0 & 1509.7 & 1429.2 & 2.41 & 4.95 & 4.59 & 0.99 & 0.1069 \\
\hline 0.6161 & 1435.6 & 1405.81371 .1 & 1499.7 & 1424.8 & 2.07 & 4.49 & 4.47 & 0.75 & 0.0962 \\
\hline 0.7001 & 1426.9 & 1403.71372 .8 & 1485.3 & 1420.2 & 1.63 & 3.79 & 1.09 & 0.47 & 0.0803 \\
\hline 0.7992 & 1412.3 & 1401.31377 .6 & 1464.9 & 1414.1 & 0.78 & 2.46 & 3.72 & 0.13 & 0.0510 \\
\hline 0.8999 & 1404.3 & 1398.91385 .6 & 1436.0 & 1406.4 & 0.38 & 1.33 & 2.26 & 0.15 & 0.0272 \\
\hline 1.0000 & 1396.8 & & & & & & & & \\
\hline \multicolumn{5}{|c|}{ Average Absolute Percentage Deviation } & 1.34 & 3.26 & 3.67 & 0.39 & \\
\hline
\end{tabular}

The deviation of the ratio $\mathrm{U}_{\exp }{ }^{2} / \mathrm{U}_{\mathrm{im}}{ }^{2}$ from unity (degree of interaction, $\alpha$ ) and its variation as a function of mole fraction of phenol is a direct measure of the nonideality of the system as a consequence of association or other type of interactions. The positive values of $\alpha$ in all the system clearly indicate the existence of strong tendency for the formation of association in mixture through hydrogen bonded complexes. The $p$-chlorophenol + DMSO mixture shows maximum in $\alpha$ while the deviation is minimum in the case of $o$-cresol + DMSO.

The DMSO is highly polar, having large dipole moment because the distance between the positive and negative charge is large. In DMSO the sulphur is bonded to oxygen atom and there is a possibility of $\mathrm{p} \pi-\mathrm{d} \pi$ bonding. In addition to a $\sigma$ bond, a $\pi$ bond may form by utilizing an unshared electron pair of oxygen and the vacant sulphur $d$-orbits. This results in a double bond, which differs from the ordinary double bond because it occurs by virtue of expansion of the valence shell to hold more than eight electrons, suggesting that $\mathrm{S}=\mathrm{O}$ bond is polar. Being strongly polarized the $\mathrm{O}-\mathrm{H}$ bond in phenols as $\mathrm{O}^{\delta-\cdots \cdots \cdots \cdots \cdots . .} \mathrm{H}^{\delta+}$, phenol[s] are able to form strong hydrogen bonds. Ali et.al ${ }^{19,20}$. Suggested the formation Hydrogen bonding between DMSO and Cyclohexanol Molecules by the excess acoustical parameter studies. The $-\mathrm{OH}$ group in phenol activates the ortho and para positions more than meta position, making the electron density high in these two positions than in the meta position.

In the $o$-cresol, the closeness of $-\mathrm{CH}_{3}$ group to $-\mathrm{OH}$ group shows the presence of two types of effect. One is the increase of electron density in the $\mathrm{O}-\mathrm{H}$ bond and the other is the steric effect. These two effects decrease the strength of intermolecular hydrogen bond formation in $o$-cresol than in meta and para cresols. In meta isomer there is no steric effect 
and the inductive effect also decreases. Due these effects the intermolecular hydrogen bond formation increases in $m$-creso ${ }^{21}$.

In $p$-cresol the inductive still decreases. But the -OH group activities the ortho and para position of the aromatic ring and deactivates the meta position. The complex formation decreases here than in meta isomer ${ }^{22,23}$.

A general survey of the Table 1 shows that $\alpha$ is positive indicates larger deviation from ideality which may be due to the formation of association in mixtures through hydrogen bonding. This result is in accordance with those of ramamurthy at al ${ }^{15,16}$. The range of percentage of deviation of ultrasonic velocity from IMR for mixtures of DMSO with phenol [s] is 2 to 4.95 where as from NR it is only 1.6 to 2.4 this shows that NR gives a satisfactory evaluation of sound velocity in binary mixtures ${ }^{24,25}$.

The degree of molecular interaction values were found to be positive in all these systems, but it becomes increasing positive in the order, $o$-cresol $<p$-cresol $<m$-cresol $<$ phenol $<p$-chlorophenol. This suggests a somewhat stronger interaction of DMSO with $p$-chlorophenol than with other phenols ${ }^{26,27}$.

The pKa (dissociation constant) values of $p$-chlorophenol, phenol, $m$-cresol, $p$-cresol and $o$-cresol are 9.20, 9.96, 10.01,10.17 and 10.20 respectively and this clearly indicates that the intermolecular hydrogen bonding is stronger in the case of $p$-chlorophenol than that of the rest of the phenols of this study ${ }^{28}$.

Possibility of formation of intermolecular bonding between the proton donor of the phenol molecules and the proton acceptor of DMSO molecules can be represented as.

(i) Phenol - DMSO

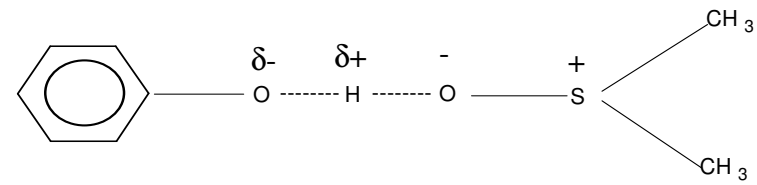

(ii) $o$ - Cresol - DMSO

(iii) $m$ - Cresol - DMSO
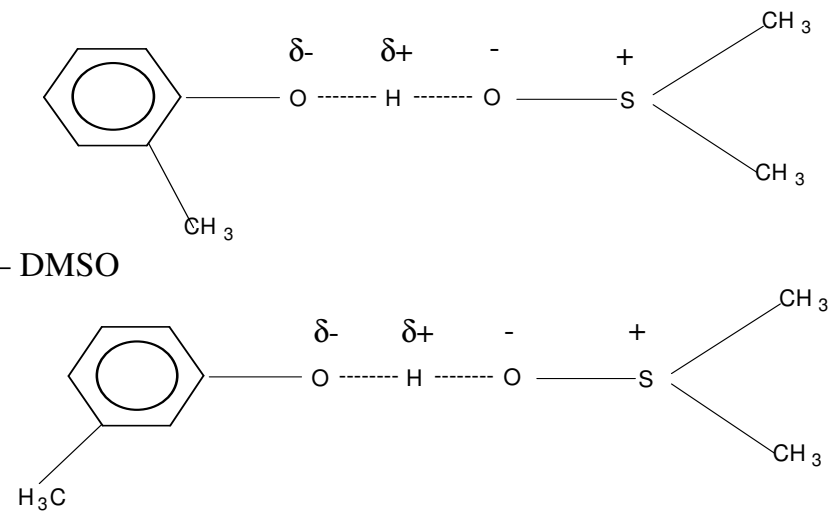

(iv) $p$-Cresol - DMSO

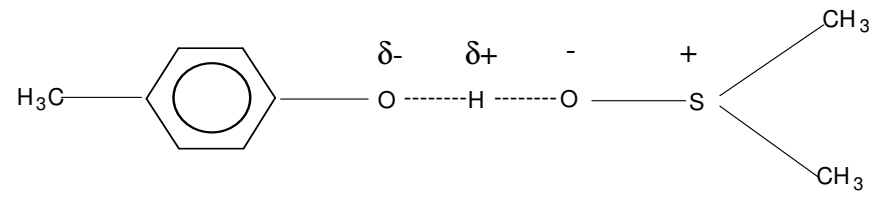


(v) $p$ - Chlorophenol-DMSO

\section{Conclusion}

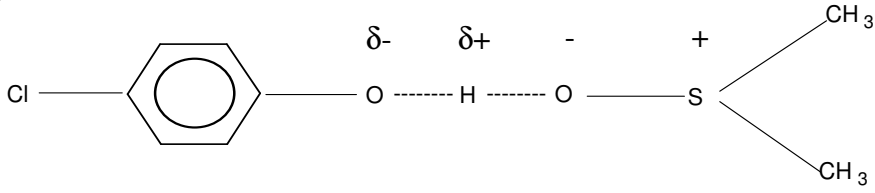

In the systems phenol[s] with DMSO, the magnitude of the degree of molecular interaction parameter values is inferred that the greatest interaction is between $p$-chlorophenol and DMSO, where as the weakest interaction between $o$-cresol and $\mathrm{DMSO}^{20,21}$. This is to be expected since the pKa value is low for $p$-chlorophenol compared to other phenols of this study. The average absolute percentage relative deviation values are also inferred the same conclusion.

\section{References}

1. Sheshagiri Rao M G, Indian J Pure Appl Phys., 1971, 9, 169.

2. Nithya A, Nithyanandham S, Mullainathan S and Rajasekaran M, E-Journal of Chemistry, 2009, 6(1),138.

3. Kannapan V and Kothai S, J Acoust Soc India, 2002, 30, 76.

4. Palani R, Saravanan S and Geetha A, Asian J Chem., 2007, 19(7), 5113.

5. Dhirendra sharma and srivastava S N, Asian J Chem., 2006, 18(4), 2756.

6. Ramaswamy K and Anbanathan D, Acustica Germany, 1981, 48, 64.

7. Nomoto O, J Phys Soc Japan, 1958, 13, 1528.

8. Van deal W and Van Geel E, Proc First International Conference Colorimetry and Thermodynamics, 1969, 555.

9. Van deal W, Thermodynamics Properties and Velocity of Sound, Burtter-worths, London, 1975, Chap II.

10. Mishra R L and Mishra R, J Acoust Soc India, 1978, 6, 32.

11. Tiwari V and Pandey J D, Indian J Pure Appl Phys., 1980, 18, 51.

12. Patil C S and Arbad B R Int J Chem Sci., 2006, 4(4), 825

13. Jacobson B, J Phys Chem., 1952, 20, 927.

14. Jacobson B, Acta Chem Scaud, 1952, 6, 1485.

15. Schaff W, Moleklara Bustik, Springer Verlag, Berlin, Chap XI and XII, 1963.

16. Nutsch - kuhnekies R, Acustica Germany, 1965, 15, 183.

17. Rajendran V, Indian J Pure Appl Phys., 1993, 39, 812.

18. Islam M R and Quadri SK, Acoust Lett., 1985, 8(10), 166.

19. Srinivasa Rao P, Subha M C S and NarayanaSwamy G, Int J Chem Sci., 2006, 4(3), 717.

20. Ali A, Nain A K, Chand D and Lal B, Phys ChemLiq., 2007, 45(1), 79.

21. Kaulgud M V and pattil K J, Indian J Pure Appl Phys., 1975, 13, 322.

22. Ramamurthy M and Sastri O S, Indian J Pure Appl Phys., 1983, 21, 579.

23. Ramamoorthy K and Varadachari P S, Indian J Pure Appl Phys., 1973, 11, 238.

24. Pandey J D Rai R D Shukla A K and Misra N, Indian J Pure Appl Phys.,1993, 31, 84.

25. Soitkar V S and Jajoo S N, Acoust Lett., 1984, 7(12), 1991.

26. Morrison R T and Boyd R N, Organic Chemistry $6{ }^{\text {th }}$ Edn., Prentice - Hall of India. (P) Ltd, New Delhi, 1992, 890.

27. Freedman J Phys Chem., 1955, 21, 1784.

28. Bhatti S S, Vivk J S and Sing D P, Acustica Germany, 1982, 50, 291. 


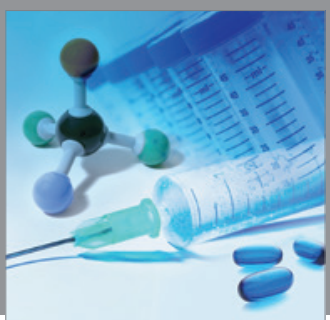

International Journal of

Medicinal Chemistry

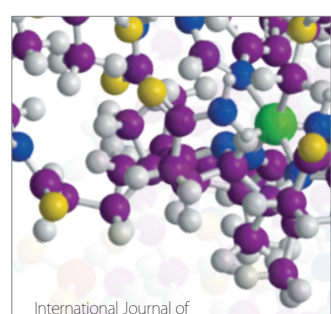

Carbohydrate Chemistry

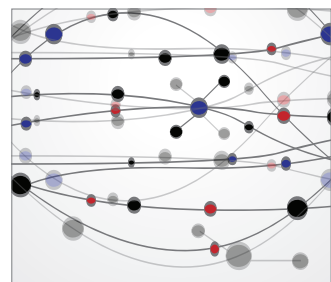

The Scientific World Journal
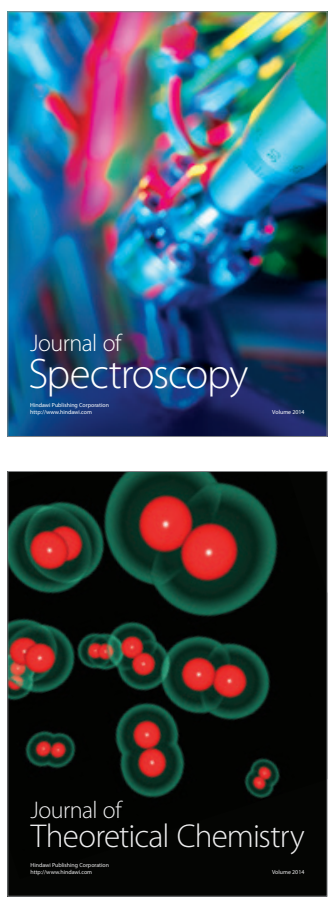
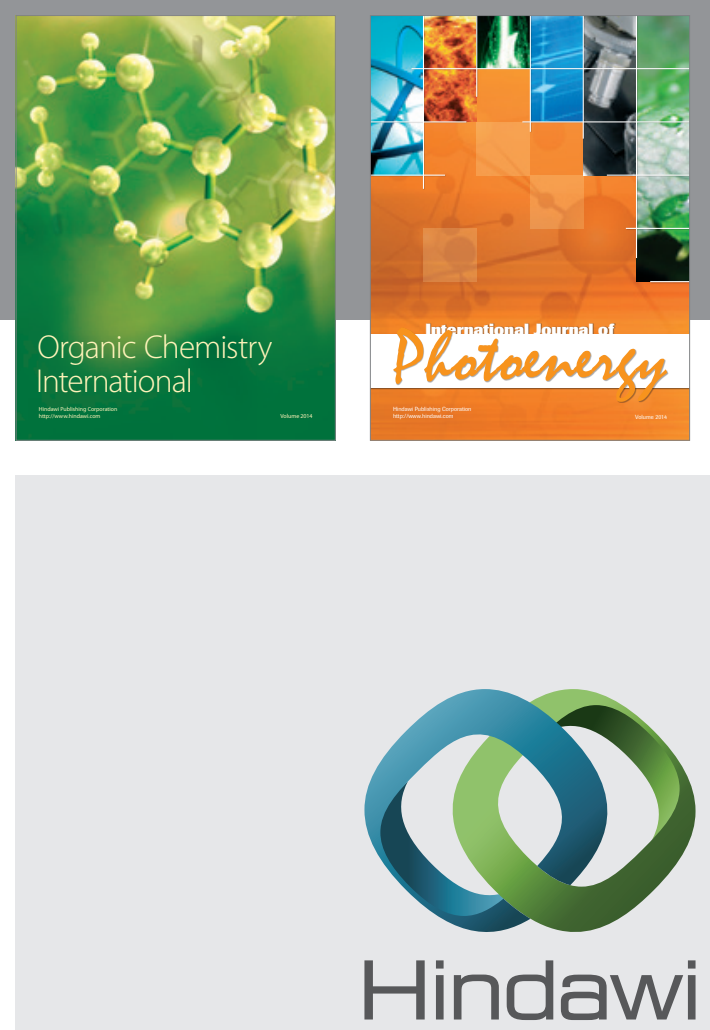

Submit your manuscripts at

http://www.hindawi.com
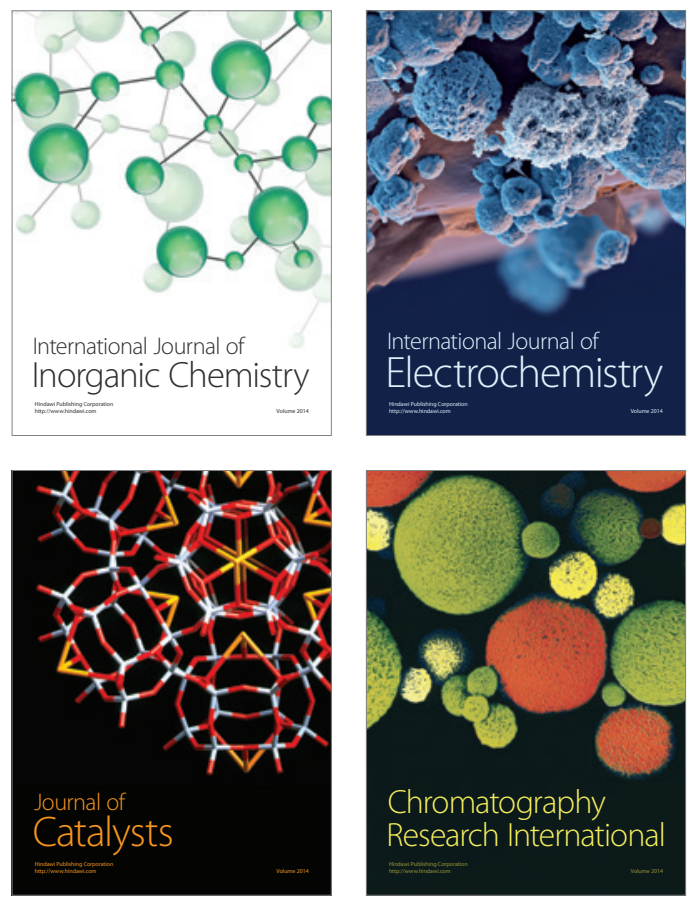
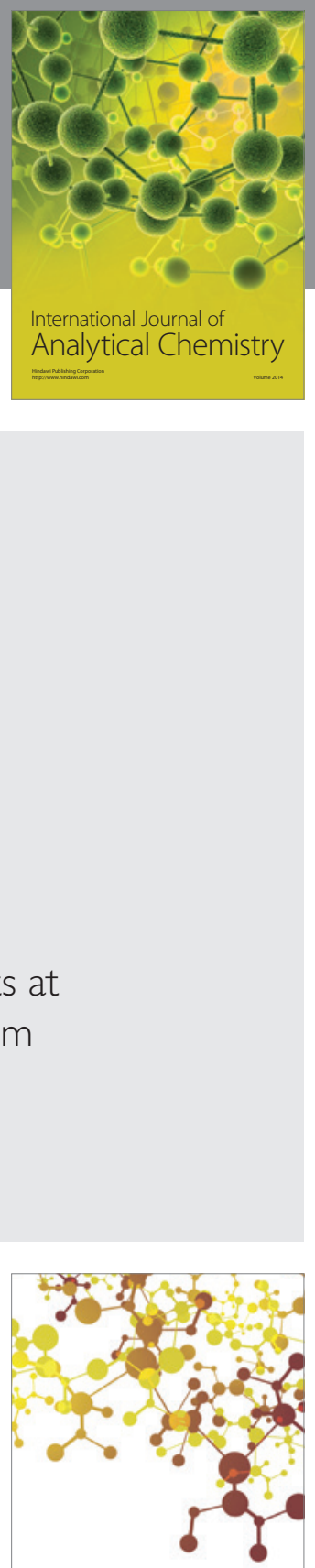

Journal of

Applied Chemistry
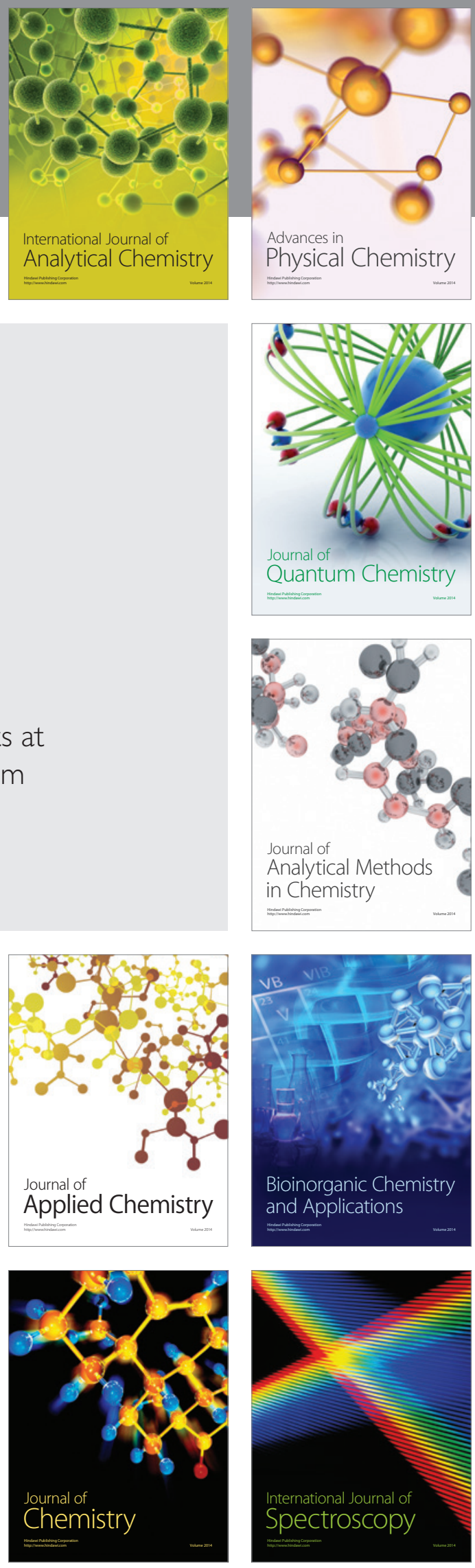